On multidimensional solitons and their legacy in contemporary Atomic, Molecular and Optical physics

This content has been downloaded from IOPscience. Please scroll down to see the full text.

2016 J. Phys. B: At. Mol. Opt. Phys. 49170502

(http://iopscience.iop.org/0953-4075/49/17/170502)

View the table of contents for this issue, or go to the journal homepage for more

Download details:

IP Address: 185.132.137.19

This content was downloaded on 02/02/2017 at 15:01

Please note that terms and conditions apply. 


\section{On multidimensional solitons and their legacy in contemporary Atomic, Molecular and Optical physics}

\author{
Boris Malomed ${ }^{1}$, \\ Lluis Torner ${ }^{2}$, \\ Frank Wise and \\ Dumitru Mihalache ${ }^{4}$ \\ ${ }^{I}$ Department of Physical \\ Electronics, School of Electrical \\ Engineering, Faculty of \\ Engineering, Tel Aviv University \\ ${ }^{2}$ ICFO-The Institute of Photonic \\ Sciences and The Barcelona \\ Institute of Science and \\ Technology \\ ${ }^{3}$ Cornell University \\ ${ }^{4}$ Institute of Physics and Nuclear \\ Engineering, Bucharest
}

(Some figures may appear in colour only in the online journal)

This viewpoint relates to an article by B A Malomed, D Mihalache, F Wise, and L Torner (2005 J. Opt. B: Quantum Semiclass. Opt. 7 R53-R72) and was published as part of a series of viewpoints celebrating 50 of the most influential papers published in the Journal of Physics series, which is celebrating its 50th anniversary.

This review article (RA) was a brief but comprehensive survey of the general area of multidimensional solitons, i.e., two- and three-dimensional (2D and 3D) modes self-trapped as a result of the competition between the linear effects of diffraction and dispersion, which tend to stretch any wave packet in spatial and temporal directions, and nonlinear self-compression of the wave field. The RA has produced an appreciable impact, having been cited 532 times (Google Scholar, as of 12 April 2016).

Multidimensional solitons draw continuously renewed interest in many branches of physics, finding especially important realizations in nonlinear photonics (optics and plasmonics) and Bose-Einstein condensates (BECs); in particular, spatiotemporal optical solitons are also known as 'light bullets'. The solitons are classified as fundamental ones, which carry no topological structure, and various topological modes, including 2D and 3D solitons with embedded vorticity, and more sophisticated 3D states, such as hopfions, i.e., vortex tori with intrinsic twist, which carry two independent topological numbers.

Unlike 1D solitons, which are normally stable, their multidimensional counterparts are vulnerable to severe instabilities. Indeed, the ubiquitous cubic selfattractive nonlinearity, which readily creates solitons, simultaneously gives rise to the critical and supercritical collapse, i.e., spontaneous formation of a singularity after a finite propagation distance or time, in the 2D and 3D geometries, respectively. The collapse destabilizes fundamental solitons, while their vortex solitons are subject to a still stronger splitting instability against perturbations breaking the axial symmetry of the vortices. Accordingly, a challenging problem is search for physically relevant settings that admit stabilization of the solitons, the settings being categorized according to the underlying stabilization mechanisms. Several generic mechanisms have been identified in the RA and developed in subsequent works: (i) the use of quadratic (second-harmonic-generating) or saturable nonlinearities, which do not lead to the collapse, and thus make fundamental solitons automatically stable, but failing to stabilize vortices; (ii) effective trapping potentials (in particular, spatially periodic ones, such as photonic lattices in optics, or optical lattices in BEC), which may stabilize 2D and 3D solitons of all types (lattice potentials create stable vortex solitons in the form of multipeak complexes, with the vorticity represented by phase shifts between adjacent peaks); (iii) competing nonlinearities, such as combinations of attractive cubic and repulsive 

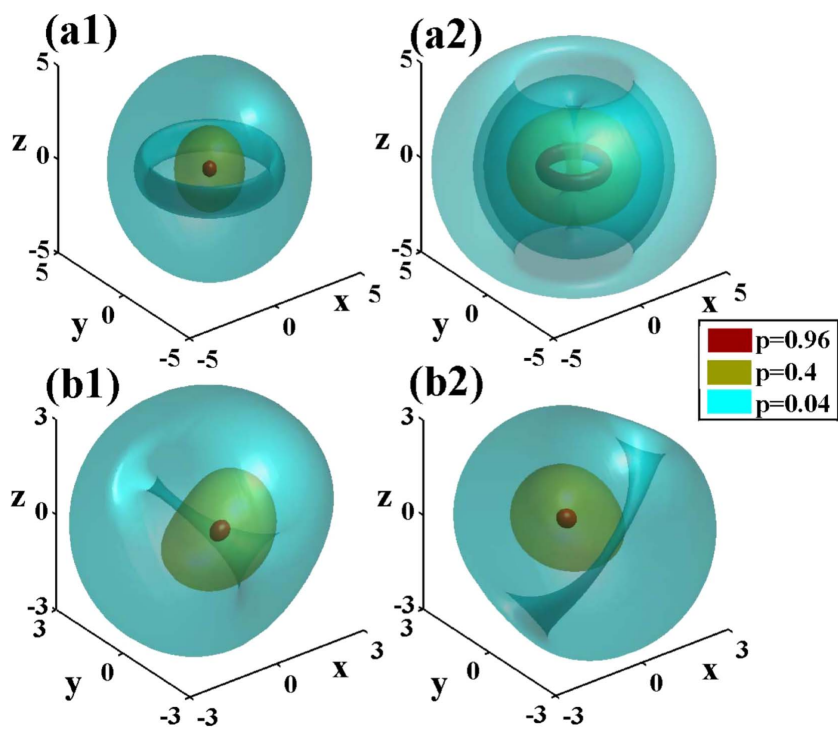

Figure 1. Examples of metastable three-dimensional solitons produced by the two-component BEC model with spin-orbit coupling, as per [12]: (a1, a2) density (p) profiles of the fundamental and vortex components; (b1, b2) the same in a soliton with the fundamental and vortex terms mixed in each component.

quintic terms, which secure partial stabilization of vortices; (iv) management techniques, which impose periodic switch of the nonlinearity between attraction and repulsion, making it possible to stabilize 2D fundamental solitons. Following the review provided in the RA, these techniques, as well as their combinations, have been developed in a large number of works, revealing many possibilities for the creation of stable solitons of different types.

An essential peculiarity of this research area, which was stressed in the RA, and remains obvious presently, is disbalance between a very large number of theoretical predictions and few experimental results. Nevertheless, in the course of 10 years since the appearance of the RA, several essential experimental findings have been published. These include, in particular, a soliton in the BEC of ${ }^{85} \mathrm{Rb}$ atoms with aspect ratio 2.5 of the trapping potential, which makes the soliton's shape close to isotropic [1]; fundamental [2] and vortex [3] 'optical bullets' created in arrays of optical fibers, which may be considered as semi-discrete media; the creation of $(2+1) \mathrm{D}$ spatial fundamental solitons in bulk optical media with a cubic-quintic [4] competing nonlinearity; 2D exciton-polariton gap solitons (supported by a lattice potential) in a microcavity [5]; direct observation of filamentation of ultrashort laser pulses in a Kerr medium in the case of negative group-velocity dispersion (which is necessary for the formation of 'bullets') [6], and the observation of a characteristic structure of the self-compressing 'bullet', composed of a high-density core and a surrounding ring pattern [7]. The creation of truly stable multidimensional vortex solitons in continuous media has not been reported yet (solitary vortices, whose limited stabilization is supported by nonlinear loss, were observed very recently [8]).

The theoretical work in the area has been developing in many directions since the publication of the RA, being, to a large extent, stimulated by results summarized in it. In particular, completely new settings allowing the creation of multidimensional solitons have been elaborated. One of them is the use of $D$ dimensional media with repulsive nonlinearity, whose local strength grows from the center to periphery, as a function of distance $r$, at any rate faster than $r^{D}$. This setting supports a variety of robust $2 \mathrm{D}$ and $3 \mathrm{D}$ solitons, including quite sophisticated ones, such as soliton gyroscopes and hopfions [9]. This direction is related to a still broader area of studies of solitons in media with effective potentials 
induced by spatial modulation of the nonlinearity [10]. Further, recent considerations of BEC with linear spin-orbit coupling between its components give rise to objects which were assumed impossible: stable solitons in free 2D [11] and 3D [12] space, supported by the attractive cubic nonlinearity, without the help of any trapping potential. They are built of mixed fundamental and vortical components, see the figure 1 . These findings help to understand the profound difference between the stabilization in 2D and 3D settings: in the former case, the system creates stable solitons as ground states, while in 3D a ground state does not exist in the presence of the supercritical collapse, the solitons being metastable modes.

\section{References}

[1] Cornish S L, Thompson S T and Wieman C E 2006 Formation of bright matter-wave solitons during the collapse of attractive Bose-Einstein condensates Phys. Rev. Lett. 96170401

[2] Minardi S et al 2010 Three-dimensional light bullets in arrays of waveguides Phys. Rev. Lett. 105263901

[3] Eilenberger F et al 2013 Observation of discrete, vortex light bullets Phys. Rev. X 3041031

[4] Falcão-Filho E L, de Araújo C B, Boudebs G, Leblond H and Skarka V 2013 Robust twodimensional spatial solitons in liquid carbon disulfide Phys. Rev. Lett. 110013901

[5] Cerda-Méndez E A, Sarkar D, Krizhanovskii D N, Gavrilov S S, Biermann K, Skolnick M S and Santos P V 2013 Exciton-polariton gap solitons in two-dimensional lattices Phys. Rev. Lett. 111146401

[6] Durand M, Jarnac A, Houard A, Liu Y, Grabielle S, Forget N, Durécu A, Couairon A and Mysyrowicz A 2013 Self-guided propagation of ultrashort laser pulses in the anomalous dispersion region of transparent solids: A new regime of filamentation Phys. Rev. Lett. 110 115003

[7] Majus D, Tamošauskas G, Gražulevičiūtè I, Garejev N, Lotti A, Couairon A, Faccio D and Dubietis A 2014 Nature of spatiotemporal light bullets in bulk Kerr media Phys. Rev. Lett. 112193901

[8] Reyna A S, Boudebs G, Malomed B A and de Araújo C B 2016 Robust self-trapping of vortex beams in a saturable optical medium Phys. Rev. A 93013840

[9] Borovkova O V, Kartashov Y V, Torner L and Malomed B A 2011 Bright solitons from defocusing nonlinearities Phys. Rev. E 84035602 (R)

Driben R, Kartashov Y V, Malomed B A, Meier T and Torner L 2014 Soliton gyroscopes in media with spatially growing repulsive nonlinearity Phys. Rev. Lett. 112020404

Kartashov Y V, Malomed B A, Shnir Y and Torner L 2014 Twisted toroidal vortex-solitons in inhomogeneous media with repulsive nonlinearity Phys. Rev. Lett. 113264101

[10] Kartashov Y V, Malomed B A and Torner L 2011 Solitons in nonlinear lattices Rev. Mod. Phys. 83247

[11] Sakaguchi H, Li B and Malomed B A 2014 Creation of two-dimensional composite solitons in spin-orbit-coupled self-attractive Bose-Einstein condensates in free space Phys. Rev. E 89 032920

[12] Zhang Y-C, Zhou Z-W, Malomed B A and Pu H 2015 Stable solitons in three dimensional free space without the ground state: self-trapped Bose-Einstein condensates with spin-orbit coupling Phys. Rev. Lett. 115253902 